\title{
Assessment of Uric Acid Level in the Saliva of Patients with Oral Lichen Planus
}

\author{
Sedigheh Bakhtiari ${ }^{\mathrm{a}}$ Parviz Toosi ${ }^{\mathrm{b}}$ Saeed Samadi ${ }^{\mathrm{a}}$ Mahin Bakhshi ${ }^{\mathrm{a}}$ \\ ${ }^{a}$ Department of Oral Medicine, Dental Faculty, and ${ }^{\mathrm{b}}$ Department of Dermatology, Shahid Beheshti University of \\ Medical Sciences, Tehran, Iran
}

\section{Keywords}

Oral lichen planus · Saliva · Uric acid

\begin{abstract}
Objective: The aim of this study was to evaluate the level of uric acid (UA) in saliva, which is considered to be an antioxidant defense biomarker against oxidative stress in patients with oral lichen planus (OLP). Subjects and Methods: In this case-control study, 25 OLP patients were included. The reticular form of OLP was verified by a clinical examination with Wickham striae, and other types (erosive, atrophic, ulcerative) were confirmed by histopathological assessment. Thirty healthy individuals matched for age and gender were selected as the control group. In both groups, the Navazesh technique was used to collect the unstimulated saliva. Then, the amount of UA was measured using a Cobas Mira autoanalyzer with a wavelength of $546 \mathrm{~nm}$. The Student $t$ test was used to analyze the data assuming a significance level at $<0.05$. Results: Of the 25 patients, the most common type of OLP was erosive $(n=11,44 \%)$, and the most common site of OLP was seen as bilateral in the buccal mucosa ( $n=12,48 \%)$. The mean level of salivary UA was significantly lower in the patients with OLP $(2.10 \pm 0.19 \mathrm{mg} / \mathrm{dL})$ in comparison with the control group $(4.80 \pm 0.29 \mathrm{mg} / \mathrm{dL} ; p<0.001)$. Conclusion:
\end{abstract}

\section{KARGER}

E-Mail karger@karger.com www.karger.com/mpp (c) 2016 S. Karger AG, Basel

Karger
Open access

This is an Open Access article licensed under the terms of the Creative Commons Attribution-NonCommercial 3.0 Unported license (CC BY-NC) (www.karger.com/OA-license), applicable to the online version of the article only. Distribution permitted for non-commercial purposes only.
In this study, OLP was associated with a decrease in UA levels in the saliva. Salivary UA as a biomarker could be used for monitoring and treating OLP.

(c) 2016 S. Karger AG, Basel

\section{Introduction}

Oral lichen planus (OLP) is considered to be a premalignant disorder and is regarded as a relatively common mucocutaneous disease [1], the exact etiology of which is still unclear [2]. Lymphocytic infiltration and keratinocyte apoptosis has been observed to likely promote the activation of a cell-mediated immune response [3]. It has been suggested that the occurrence of OLP could be triggered by imbalances among the antioxidant stress markers in biological body fluids, and thus could play an important role in the pathogenesis of OLP transformation $[4,5]$. An oxidative metabolism can lead to oxidative stress as a harmful byproduct and molecular destruction in living systems, which are subsequently involved in numerous processes, such as aging, mutagenesis, and a series of pathological events [6].

Oxidative stress in OLP releases molecules consisting of granzymes that may result in local tissue damage in 
Table 1. Types of OLP in the case subjects, $n$ (\%)

\begin{tabular}{lc}
\hline Reticular & $7(28)$ \\
Erosive & $11(44)$ \\
Atrophic & $4(16)$ \\
Ulcerative & $3(12)$ \\
Total & $25(100)$ \\
\hline
\end{tabular}

Table 2. The location of OLP in the case subjects, $n$ (\%)

\begin{tabular}{lr}
\hline Buccal mucosa (both sides) & $12(8)$ \\
Buccal mucosa (1 side) & $4(6)$ \\
Buccal mucosa (both sides) + tongue & $4(6)$ \\
Buccal mucosa (both sides) + vestibular & $1(4)$ \\
Tongue & $1(4)$ \\
Vestibular mucosa & $1(4)$ \\
Mandibular ridge & $1(4)$ \\
Lip & $1(4)$ \\
\hline Total & $25(100)$ \\
\hline
\end{tabular}

the effectors [7]. Antioxidants can defend against oxidative stress and are present in mammalian cells, such enzymes as superoxide dismutase, catalase, and glutathione peroxidase, as well as nonenzymatic antioxidants, including melatonin, uric acid (UA), and vitamins A and $\mathrm{E}[8,9]$.

As a scavenger of oxyradicals and chelator of metal ions, UA is known to be an important antioxidant that is predominant in plasma $[10,11]$. In the current study, saliva testing was utilized since a linear relationship exists between its UA concentration and serum. It is considered to be a helpful noninvasive fluid for the monitoring of biomarkers $[12,13]$. However, limited data are found in the literature about the UA level of saliva in OLP. Thus, to our knowledge, this study is the first such investigation to be conducted in Iran.

\section{Subjects and Methods}

This case-control study was performed on Iranian patients admitted to the Oral Medicine Department and also enrolled healthy volunteers. Informed consents were obtained along with ethical committee approval.

\section{Selection of Patients}

Twenty-five patients with OLP (cases), including 15 women and 10 men, and 30 healthy subjects (controls), including 20 women and 10 men, were compared. The reticular form of OLP was verified by a clinical examination with Wickham striae, while oth- er types (erosive, atrophic, ulcerative) were confirmed by histopathological assessment. Histopathological confirmation was carried out according to the WHO criteria [14].

The exclusion criteria were the presence of any systemic disease (gout, diabetes, hypertension, thyroid disease, heart disease, kidney disease, hepatitis C), alcohol consumption, smoking, the consumption of drugs that increase UA (e.g. antidiuretics), immunosuppressive drugs, nonsteroid anti-inflammatory drugs, systemic or topical corticosteroids, vitamin supplements over the past month, a history of surgery or trauma in the past month, and having been treated for lichen planus or currently undergoing treatment [15].

\section{Collection of Saliva}

The patients refrained from eating and drinking for $2 \mathrm{~h}$ prior to sampling. Then, between 9 and 12 a.m., $2 \mathrm{~mL}$ of unstimulated saliva was collected from both groups through a spitting method [16]. The samples were immediately stored at $-70^{\circ} \mathrm{C}$. Centrifugation (Behdad Chemical Co., Tehran, Iran) of the test samples at $1,008 \mathrm{~g}$ was then performed for $10 \mathrm{~min}$ to separate the supernatant in order to measure the UA antioxidant using a Pars Azmun kit made in Iran. The results were recorded with the help of a trained impartial person.

\section{UA Antioxidant Measurement}

The level of UA activity in the saliva was determined based on the tooth colorimetric method (N-ethyl-N-[2-hydroxy3-sulfopropyl]-3-methylaniline). A sample of $100 \mathrm{~mm}$ phosphate buffer of the reagent No. 1, pH 7.1, 1 mM TOOS, and $1 \mathrm{kU} / \mathrm{L}$ ascorbate oxidase was added to $20 \mu \mathrm{L}$ of each sample. Incubation of the reaction mixture was conducted for $5 \mathrm{~min}$ at $37^{\circ} \mathrm{C}$. Then, the samples were combined with $100 \mathrm{mM}$ phosphate buffer of reagent No. 2, pH 7.0, 0.3 mM 4-aminoantipyrine, $0.1 \mu \mathrm{M} \mathrm{K} \mathrm{K}_{4}(\mathrm{Fe}[\mathrm{CN}] 6), 1 \mathrm{kU} / \mathrm{L}$ peroxidase, and $50 \mathrm{kU}$ uricase, and incubated for $5 \mathrm{~min}$ at $37^{\circ} \mathrm{C}$. The amount of UA was measured by photometry using a Cobas Mira autoanalyzer at a wavelength of $546 \mathrm{~nm}$ [17].

\section{Statistical Analysis}

SPSS software v.18.0 for Windows (SPSS, Chicago, IL, USA) was used. The Student $t$ test was used to analyze the data at a significance level of $p<0.05$.

\section{Results}

The mean ages of the patients with OLP and controls were $46 \pm 2.33$ and $36 \pm 1.18$ years, respectively. Seven (28\%) patients had reticular OLP that was confirmed clinically, and 18 (72\%) patients were diagnosed with erosive, atrophic, or ulcerative OLP according to biopsy assessment. Among the patients, the most common type of OLP was erosive $(11,44 \%)$, and the most frequent site of OLP was seen as bilateral in the buccal mucosa (12, $48 \%$ ). The frequency and number of OLP types and their locations in the oral mucosa are presented in Tables 1 and 2. 


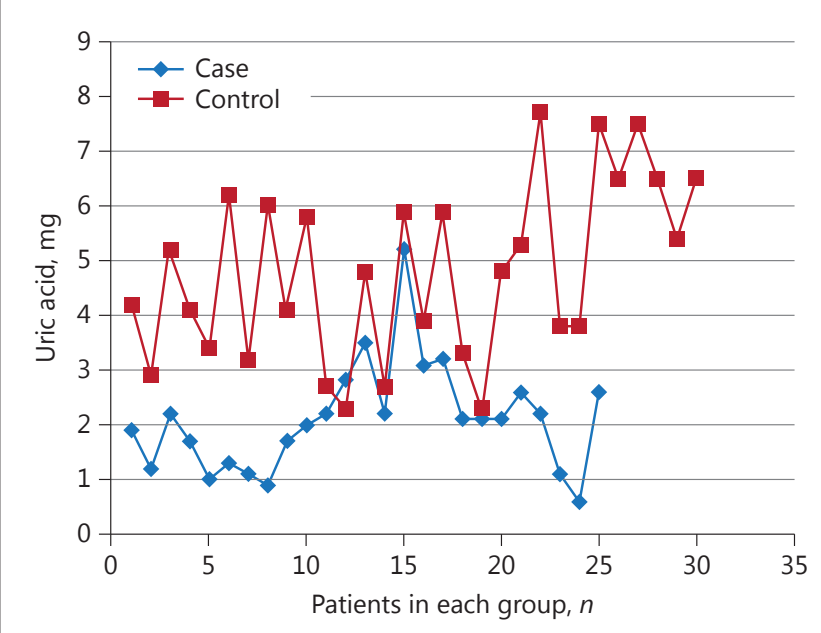

Fig. 1. Salivary UA in the cases and controls.

The mean salivary UA in the case group $(2.10 \pm 0.19$ $\mathrm{mg} / \mathrm{dL}$ ) was significantly lower than that in the control group $(4.80 \pm 0.29 \mathrm{mg} / \mathrm{dL} ; \mathrm{p}<0.001)$. The comparison of salivary UA in the cases and controls is presented as a scatter plot in Figure 1.

\section{Discussion}

In this study, the level of salivary UA in the OLP patients was lower than in the control subjects. This finding confirmed that of Miricescu et al. [18], who reported lower UA levels in unstimulated saliva from OLP patients compared to the control group. Of equal importance, lower blood UA levels were found in patients with cutaneous lichen planus in comparison with the control group in the study by Chakraborti et al. [19]. Moreover, Miricescu et al. [18] detected lower UA and total antioxidant capacity levels in the saliva of smokers and OLP patients involved in periodontitis compared to the control group.

Regarding the monitoring of some antioxidants, such as UA, bilirubin, glutathione peroxidase, and vitamin C in OLP patients who also presented cutaneous lesions, Barikbin et al. [15] found only decreased levels of vitamin $\mathrm{C}$ in the blood compared to the control group. In our study, in which we used saliva instead of blood, only UA demonstrated a significant decrease.

OLP occurrence has been suggested to be triggered by an enhanced oxidative stress and antioxidant defense sys- tem imbalance in biological body fluids, thus reflecting the pathogenesis of OLP transformation. As a scavenger of oxyradicals and chelator of metal ions, UA is known to be an important antioxidant. It is also a predominant antioxidant in plasma $[10,11]$.

UA is an end product of purine metabolism and is produced in mammalian systems. It contaminates free radical substances through the inhibition of endothelial function under conditions of oxidative stress inside the cell in which glutathione is discharged [20]. It can further inhibit the ability of exogenous peroxynitrite to uncouple endothelial nitric oxide (NO) synthase. New compounds, such as peroxynitrite, are formed to further damage cells through the interface of $\mathrm{NO}$ with such oxygen radicals as superoxide [21]. Nevertheless, intracellular NO levels can be reduced by high UA concentrations [10].

It is thus suggested that an optimal strengthening of antioxidant defense be administered at the UA level as a treatment guideline since a major factor in the etiopathogenesis of lichen planus is deemed to be induced by free radical damage. Our findings were indicative of a reduced antioxidant UA level in OLP patients, and further investigation is warranted on a greater number of patients to assess other antioxidants and oxidative stress biomarkers for the treatment of oxidative stress in OLP, which we have shown to be likely associated with the depletion of salivary UA levels.

\section{Conclusion}

Our study showed that OLP was associated with decreased UA levels in saliva. UA could be a useful antioxidant biomarker for monitoring and guiding the treatment strategy in OLP. Hence, further studies are needed to compare and monitor the level of UA in treated patients over time.

\section{Acknowledgements}

This study was based on an undergraduate thesis. The authors wish to thank the Research Center of Shahid Beheshti University of Medical Sciences and the Dermatology Department in Shohada Hospital, Tehran, Iran, for their beneficial support, and Dr. Fateme Mashhadi Abbas for the assessment of biopsied specimens.

\section{Disclosure Statement}

The authors have no conflicts of interest to declare. 


\section{References}

1 Glick M, Feagans WM: Burket's Oral Medicine, ed 12. Shelton, People's Medical, 2015, p 109.

2 Munde AD, Karle RR, Wankhede PK, et al: Demographic and clinical profile of oral lichen planus: a retrospective study. Contemp Clin Dent 2013;4:181-185.

3 Thornhill MH: Immune mechanisms in oral lichen planus. Acta Odontol Scand 2001; 59: 174-177.

4 Ergun S, Troşala SC, Warnakulasuriya S, et al: Evaluation of oxidative stress and antioxidant profile in patients with oral lichen planus. J Oral Pathol Med 2011;40:286-293.

5 Aly DG, Shahin RS: Oxidative stress in lichen planus. Acta Dermatovenerol Alp Pannonica Adriat 2010;19:3-11.

6 Finkel T, Holbrook NJ: Oxidants, oxidative stress and the biology of ageing. Nature 2000; 408:239-247.

7 ScrobotăI I, Mocan T, Cătoi C, et al: Histopathological aspects and local implications of oxidative stress in patients with oral lichenplanus. Rom J Morphol Embryol 2011;52: 1305-1309.

8 Nagler RM, Klein I, Zarzhevsky N, et al: Characterization of the differentiated antioxidant profile of human saliva. Free Radic Biol Med 2002;32:268-277.
9 Momen-Beitollahi J, Mansourian A, MomenHeravi F, et al: Assessment of salivary and serum antioxidant status in patients with recurrent aphthous stomatitis. Med Oral Patol Oral Cir Bucal 2010;15:557-561.

10 Gersch C, Palii SP, Kim KM, et al: Inactivation of nitric oxide by uric acid. Nucleosides $\mathrm{Nu}$ cleotides Nucleic Acids 2008;27:967-978.

11 Kutzing MK, Firestein BL: Altered uric acid levels and disease states. J Pharmacol Exp Ther 2008;324:1-7.

12 Soukup M, Biesiada I, Henderson A, et al: Salivary uric acid as a noninvasive biomarker of metabolic syndrome. Diabetol Metab Syndr 2012;4:14.

13 Bakhtiari S, Toosi P, Dolati F, et al: Evaluation of salivary secretor status of blood group antigens in patients with oral lichen planus. Med Princ Pract 2016;25:266-269.

14 Mravak-Stipetić M, Lončar-Brzak B, BakaleHodak I, et al: Clinic pathologic correlation of oral lichen planus and oral lichenoid lesions: a preliminary study. Sci World J 2014;2014: 746874 .
15 Barikbin B, Yousefi M, Rahimi H, et al: Antioxidant status in patients with lichen planus. Clin Exp Dermatol 2011;36:851-854.

16 Navazesh M; ADA Council on Scientific Affairs and Division of Science: How can oral health care providers determine if patients have dry mouth? J Am Dent Assoc 2003; 134: 613-620.

17 Bablok W, Passing H, Bender R, et al: A general regression procedure for method transformation: application of linear regression procedures for method comparison studies in clinical chemistry, part III. J Clin Chem Clin Biochem 1988;26:783-790.

18 Miricescu D, Greabu M, Totan A, et al: The antioxidant potential of saliva: clinical significance in oral diseases. Molecules 2011;4:5.

19 Chakraborti G, Biswas R, Chakraborti S, et al: Altered serum uric acid level in lichen planus patients. Indian J Dermatol 2014;59:558-561.

20 Shamsi FA, Hadi SM: Photoinduction of strand scission in DNA by uric acid and Cu(II). Free Radic Biol Med 1995;19:189-196.

21 Panjwani S, Bagewadi A, Keluskar V, et al: Estimation and comparison of levels of salivary nitric oxide in patients with oral lichen planus and controls. Int J Prev Med 2013;4:710-714. 